\title{
Ultrasound-guided supraclavicular cannulation of the brachiocephalic vein may reduce central line-associated bloodstream infection in preterm infants
}

\author{
Ignacio Oulego-Erroz ${ }^{1,2,3}$ (1) Alba Fernández-García ${ }^{4}$ - Beatriz Álvarez-Juan ${ }^{4} \cdot$ Sandra Terroba-Seara ${ }^{4}$. \\ Paula Alonso Quintela ${ }^{3,4} \cdot$ Antonio Rodríguez-Núñez ${ }^{5}$
}

Received: 27 March 2020 / Revised: 17 April 2020 / Accepted: 22 April 2020 / Published online: 7 May 2020

(C) Springer-Verlag GmbH Germany, part of Springer Nature 2020

\begin{abstract}
The objective of this study was to assess the risk of central line-associated bloodstream infection (CLABSI) of ultrasound (US)guided cannulation of the brachiocephalic vein $(\mathrm{BCV})$ compared to standard epicutaneous cava catheters (ECCs) in preterm infants. This was a retrospective cohort study in preterm infants with a birth weight of less than $1500 \mathrm{~g}$. Each BCV catheter was matched 1:3 with ECCs according to sex, birth weight, and year of insertion. The main outcome was the CLABSI density rate per 1000 days. Secondary outcomes included CLABSI episodes, CLABSI episodes per infant, and CLABSI/death. A multivariate Cox regression analysis was performed to assess whether the type of catheter (ECC vs. BCV) was associated with CLABSI risk. Ninety-six catheters (21 BCVs and $75 \mathrm{ECCs}$ ) in 79 infants were included (993 catheter days). BCV catheters were associated with a reduced CLABSI density rate compared to ECCs (3.05/1000 days vs $21.1 / 1000$ days; $p<0.001)$. ECCs were associated with increased CLABSI risk compared to BCV catheters in multivariate analysis (hazard ratio 36; (95\% CI, 2.5-511); $p=0.008$ ).

Conclusion: US-guided supraclavicular cannulation of the $\mathrm{BCV}$ was associated with a reduced risk of CLABSI compared to ECCs. This finding deserves further multicenter research.
\end{abstract}

What is Known:

- An epicutaneous-cava catheter (ECC) is commonly used in preterm infants for routine care (eg. delivery of nutrition and antibiotics) but this device may not suffice in infants who need high-intensity care (multiple drugs, hemodynamic monitoring, fluid resuscitation etc.).

- Ultrasound-guided brachiocephalic vein (BCV) catheterization has shown a high success rate and few immediate complications in neonates and small infants but it has never been compared to standard ECCs.

What is New:

- When the operator in properly trained, US guided cannulation of the BCV in preterm infants is feasible, safe and may reduce the risk of CLABSI compared to standard ECCs.

- This fact may expand the use of BCV catheters in selected high-risk preterm infants who need a large bore venous access.

Keywords Preterm $\cdot$ Central line-associated bloodstream infection $\cdot$ Ultrasound $\cdot$ Brachiocephalic vein $\cdot$ Epicutaneous cava catheter

Communicated by Daniele De Luca

Ignacio Oulego-Erroz

ignacio.oulego@gmail.com

Alba Fernández-García albafg291@gmail.com

Beatriz Álvarez-Juan beatriz.alvarez.juan@gmail.com

Paula Alonso Quintela paulaalonsoquintela@gmail.com

Antonio Rodríguez-Núñez

Antonio.rodriguez.nunez@sergas.es
1 Pediatric Intensive Care Unit, Complejo Asistencial Universitario de León, León, Spain

2 Working Group on Bedside Ultrasound of the Spanish Society of Pediatric Intensive Care (SECIP), León, Spain

3 IBIOMED - Biomedicine Institute of León, University of León, León, Spain

4 Neonatal Intensive Care Unit, Complejo Asistencial Universitario de León, León, Spain

5 Pediatric Critical, Intermediate and Palliative Care Section, Hospital Clínico Universitario de Santiago, Research Institute of Santiago (IDIS), Santiago, Spain 


\section{Introduction}

Insertion of a central venous catheter (CVC) is commonly used in preterm infants admitted to the neonatal intensive care unit (NICU). The two most common vein-access techniques in this population are umbilical vein catheters (UVCs) during the first days of life and peripherally inserted central catheters (ECCs) thereafter [1].

Occasionally, for the sickest preterm infants, large-bore percutaneous CVC may be needed for fluid resuscitation, for drug administration, for hemodynamic monitoring, or after peripheral vein exhaustion $[2,3]$. A relatively new technique consisting of US-guided cannulation of the brachiocephalic vein $(\mathrm{BCV})$ from a supraclavicular approach has been associated with a high success rate and few complications in infants and neonates $[4,5]$. However, no study to date has assessed the potential advantages of this approach in terms of central line-associated bloodstream infection (CLABSI) in neonates. In our unit, we have implemented US-guided insertion of large-bore percutaneous $\mathrm{CVCs}$ through the $\mathrm{BCV}$ in selected sick neonates and small infants, with good results [6]. In this study, we tested the hypothesis that US-guided BCV cannulation has a lower risk of CLABSI than standard ECCs in preterm infants.

\section{Methods}

\section{Design and setting}

This is a retrospective cohort study of preterm infants admitted to a tertiary level-3B NICU between January 1, 2016, and December 31, 2019.

\section{Participants}

Very-low-birth-weight infants $(<1500 \mathrm{~g})$ under 34 weeks of gestational age in whom US-guided BCV cannulation or ECC insertion was performed were eligible. $\mathrm{BCV}$ and $\mathrm{ECC}$ procedures were matched 1:3 according to the patient's birth weight, sex, and year of insertion. Infants with CVCs in place for less than 6 hours, who died within the first 48 hours of life or for whom data records were incomplete, were excluded from the analysis. Infants were followed until discharge from the Neonatal Unit.

\section{CVC indications, insertion technique, and maintenance policy}

In this study, the exposure variable was the type of CVC inserted (exposed: BCV; unexposed: ECC). In our unit, preterm infants below 33 weeks of gestation receive a UVC at admission to the NICU as a general rule. In general, UVCs are withdrawn electively as soon as possible after the 4th day of life (DOL) and always before the 10th DOL and substituted by an ECC or a peripheral cannula depending on the patient's clinical condition and the ongoing need for a CVC. ECCs are inserted through forearm veins without US guidance under sterile conditions and advanced to the distal third portion of the superior vena cava (SVC). CVC tip position is checked by a chest X-ray. A noncentral catheter tip position at the intrathoracic subclavian or brachiocephalic vein is sometimes accepted. During the study period, bilumen 2 French polyurethane ECC lines (Nutriline Twinflow ${ }^{\circledR}$, Vygon; Aachen, Germany) were used. Non-pharmacological sedation methods (contention) and 24\% sucrose solution are routinely used. Only neonatal nurses are qualified to place ECCs. Neonatal nurses work full time in the neonatal unit. They must have a 2-year experience in pediatric nursing (including a 6month rotation in the neonatal unit) and a supervised training by senior staff in ECC placement before they are qualified to perform the procedure autonomously.

Selected infants are considered candidates for percutaneous insertion of a large-bore CVC. Indications include severe hemodynamic instability (e.g., pulmonary hypertension, septic shock), high-risk surgery (e.g., necrotizing enterocolitis (NEC) surgery or patent ductus arteriosus (PDA) ligation), the need for multiple drug infusions, or exhausted peripheral veins. From 2014, we implemented in-plane US-guided supraclavicular BCV cannulation as the technique of choice for critically infants and neonates under $5 \mathrm{~kg}$. In this study, all procedures were performed by a pediatrician with 10 years of experience in US-guided CVC placement in children (including more than 150 procedures using the supraclavicular $\mathrm{BCV}$ approach). A portable US machine (Sonosite Turbo, Fujifilm Japan) equipped with a linear 6-13-MHz transducer (L25x linear probe) was used. Prior to the procedure, the left and right $\mathrm{BCV}$ diameters were assessed. The optimal catheter size was selected by keeping a catheter:vein ratio of $\leq 1: 3$ to prevent blood flow obstruction and thrombosis [7, 8]. Doublelumen 3- and 4-French short $(5-6 \mathrm{~cm})$, noncoated catheters were used in all instances (Multicath 2®, Vygon; Aachen, Germany and Standard Unimpregnated CVC, Cookmedical; Bloomington, IN, USA) depending on the size of the vein. All procedures were performed using the conventional Seldinger technique under sterile conditions and pharmacological sedation. Nonintubated preterms were sedated with midazolam $(0.05-0.1 \mathrm{mg} / \mathrm{kg})$ and ketamine $(0.5-1 \mathrm{mg} / \mathrm{kg})$ while fentanyl $(1 \mathrm{mcg} / \mathrm{kg})$ was used in infants under invasive mechanical ventilation. Mepivacaine $2 \%$ was used for local anesthesia of the skin (maximal dose $5 \mathrm{mg}$ ). The cannulation side that provided a better long-axis view of the $\mathrm{BCV}$ was selected, and the patient was positioned with the neck extended and rotated $45^{\circ}$ opposite to that side. For patients under mechanical ventilation, the ventilator settings were not manipulated during cannulation. The linear transducer was positioned in the 
supraclavicular area to obtain a long-axis view of the $\mathrm{BCV}$ and subclavian vein. The subclavian artery and the underlying lung pleura were identified before puncture. A 22-gauge echogenic introducer needle attached to a syringe was used to puncture the vein. The needle was inserted in plane from the lateral aspect of the transducer and directed under real-time visualization to the $\mathrm{BCV}$. Blood was aspirated to confirm intravascular positioning, and a straight soft-tipped 0.018 -in. guidewire was advanced into the vessel and checked by US visualization. The CVC tip was navigated and adequacy of tip position was assessed by real-time cardiac and vascular US using a predefined protocol [9]. A noncentral catheter tip position was not allowed. The procedure was completed as usual and the catheter was fixed using $5-0$ non-absorbable surgical suture.

The same maintenance policy is invoked for all CVCs. Catheters are always manipulated under sterile conditions, avoiding (if possible) 3-way stop-lockers. A unique port is reserved for parenteral nutrition. Low-dose heparin $(0.5 \mathrm{U} /$ $\mathrm{kg} / \mathrm{h}$ ) infusion is used to maintain catheter patency [10]. Transparent dressings are used and changed every 7 days or when blood is present or the dressing is disrupted. The catheter insertion site is assessed daily for signs of infection such as redness, swelling, or purulent discharge. When CLABSI is suspected, peripheral blood cultures with or without paired central line blood cultures are obtained, and empiric antibiotics (vancomycin plus amikacin) are started. The catheter is withdrawn if CLABSI is confirmed or if the infant deteriorates, except in cases of coagulase-negative Staphylococcus (CoNS), in which the CVC is initially kept in place under close monitoring. Surveillance blood or catheter tip cultures are not performed for asymptomatic patients.

\section{Measurements and outcomes}

Perinatal data, clinical characteristics, and catheterrelated outcomes were prospectively recorded according to a nationwide registry (SEN1500 network) and the European healthcare-associated infections surveillance system (NEO-KISS network) [11, 12]. Periprocedural data (first attempt and overall success, number of puncture attempts, immediate complications, etc.) were prospectively recorded as a part of an ongoing local registry of percutaneous CVCs.

The main outcome of this study was the CLABSI rate per 1000 days of catheter use. Secondary outcomes included CLABSI episodes per infant (no. of CLABSI episodes/no. of infants), CLABSI episodes per catheter (no. of CLABSI episodes/no. of catheters) and combined CLABSI per infant or death. CLABSI was defined as the presence of clinical symptoms of sepsis (at least two of the following: fever, hypothermia, apnea, bradycardia, respiratory distress, poor peripheral perfusion, or hypotension) with elevated acute-phase reactants (C-reactive protein $\geq 10 \mathrm{mg} / \mathrm{dl}$ and $/$ or procalcitonin $>3 \mathrm{ng} / \mathrm{ml}$ beyond the $72 \mathrm{~h}$ of life) [13] and a positive blood or catheter tip culture in the first $48 \mathrm{~h}$ after catheter insertion until $48 \mathrm{~h}$ after catheter withdrawal. In the case of CoNS, $\geq 2$ positive blood cultures drawn at different time points were necessary to diagnose CLABSI according to the current CDC/NHSN surveillance definition [14]. With the aim of being pragmatic, if $\geq 2$ peripheral blood cultures could not be obtained, CoNS-CLABSI was considered proven if blood and catheter tip cultures were positive for a CoNS with an identical antibiogram.

\section{Statistical analysis}

Categorical variables are summarized as numbers (percentages), and continuous variables are summarized as medians (interquartile ranges) and means (standard deviations). The chi-square test was employed for comparisons of categorical variables; nonparametric tests (the Mann-Whitney $U$ test) were used for comparisons of continuous variables. The occurrence of CLABSI was estimated with the Kaplan-Meier product-limit estimator and log-rank test. A multivariate Cox regression analysis was performed to assess whether CVC type (ECC vs BCV) is an independent risk factor for CLABSI. Potential risk factors for CLABSI with $p<0.1$ as the entry criterion were selected by the step-forward method for the multivariate model, with the aim of controlling confounders. Assuming that infants have the same chance to receive either CVC type (BCV or ECC) and a matched design with an exposed:unexposed ratio of 1:3, a sample size of 20 $\mathrm{BCV}$ (and $60 \mathrm{ECCs}$ ) will provide a power of 0.8 (alpha error 0.05 ) to detect a 3-fold decrease in the CLABSI density rate. With a planned insertion of $5 \mathrm{BCV}$ catheters/year, we chose a time window of 4 years to extract data from our cohort to convey with our sample size calculation. A $p$ value $<0.05$ was considered statistically significant. The SPSS (IBM Corp., Arkmonk, N.Y., USA) statistical package version 22 was used.

\section{Ethical aspects}

The study protocol was reviewed and approved by the local IRB. Parents gave informed consent for data entry into the SEN1500 and NEO-KISS network records. Given the retrospective nature of our study, the IRB waived the need for informed consent for the present analysis.

\section{Results}

Ninety-six CVCs (21 BCV catheters and 75 ECCs) in 79 infants were included (Table 1). Another 38 VLBW infants 
Table 1 Clinical characteristics of the study groups

\begin{tabular}{|c|c|c|c|}
\hline Clinical characteristics & $\operatorname{BCV}(n=21)$ & $\mathrm{ECC}(n=58)$ & $p$ \\
\hline Sex (female) & $8(38.1)$ & $19(32.7)$ & 0.659 \\
\hline Gestational age (weeks) & $27(26+1-27+1)$ & $27+6(26+6-29+4)$ & 0.002 \\
\hline Birth weight (grams) & $980(815-1090)$ & $990(786-1172)$ & 0.731 \\
\hline Cesarean delivery & $15(71.4)$ & $38(65.5)$ & 0.621 \\
\hline Twin pregnancy & $3(14.3)$ & $14(24.1)$ & 0.341 \\
\hline Maternal chorioamnionitis & $4(19)$ & $7(12.1)$ & 0.429 \\
\hline Antenatal steroids & $18(81.8)$ & $55(94.5)$ & 0.177 \\
\hline Apgar 5 min & $8(6-8)$ & $8(7-9)$ & 0.021 \\
\hline CRIB score $12 \mathrm{~h}$ & $4(2.5-6)$ & $2.5(1-6)$ & 0.392 \\
\hline Perinatal risk factors of infection & $15(71.4)$ & $45(77.6)$ & 0.484 \\
\hline RDS (surfactant replacement) & $15(71.4)$ & $29(50)$ & 0.090 \\
\hline Invasive mechanical ventilation (days) & $6(3.5-10)$ & $6(3.5-10)$ & 0.015 \\
\hline Inotropics/vasopressors & $18(85.7)$ & $33(56.8)$ & 0.027 \\
\hline Blood transfusion & $18(85.7)$ & $35(60.3)$ & 0.034 \\
\hline Retinopathy & $3(14 \%)$ & $5(8.6)$ & 0.476 \\
\hline Grade III/IV IVH & $2(9.5)$ & $6(10.3)$ & 0.915 \\
\hline Hemodynamically significant PDA & $14(66.6)$ & $26(44.8)$ & 0.086 \\
\hline PDA surgery & $5(23.8)$ & $0(0)$ & 0.005 \\
\hline Necrotizing enterocolitis & $7(33 \%)$ & $7(12.1)$ & 0.029 \\
\hline Bronchopulmonary dysplasia* & $12(57.1)$ & $29(50)$ & 0.575 \\
\hline Death & $2(9.5)$ & $5(8.6)$ & 0.767 \\
\hline \multicolumn{4}{|l|}{ Cause of death: } \\
\hline -Adequacy of life support & 1 & 3 & \\
\hline -Septic shock/NEC & 0 & 2 & - \\
\hline -Respiratory failure & 1 & 0 & \\
\hline
\end{tabular}

Abbreviations: CRIB clinical risk index for babies; IVH intraventricular hemorrhage; $N E C$ necrotizing enterocolitis; $P D A$ patent ductus arteriosus; $R D S$ respiratory distress syndrome. *Refers to oxygen and/or ventilator dependency at 36 weeks of postmenstrual age (moderate-severe BPD) were initially assessed for eligibility during the study period but excluded for several reasons (Fig. 1). In one infant, BCV cannulation failed, and an internal jugular vein catheter was inserted; he was also prospectively excluded. All patients received a UVC for a median of 8 days [6-8] before ECC or $\mathrm{BCV}$ insertion. Preterm infants in whom US-guided BCV catheters were inserted had a lower gestational age and suffered from more morbidities (mechanical ventilation days,
Fig. 1 Flow chart of the study. Abbreviations: $\mathrm{BCV}$, brachiocephalic vein; ECC, peripherally inserted central catheter

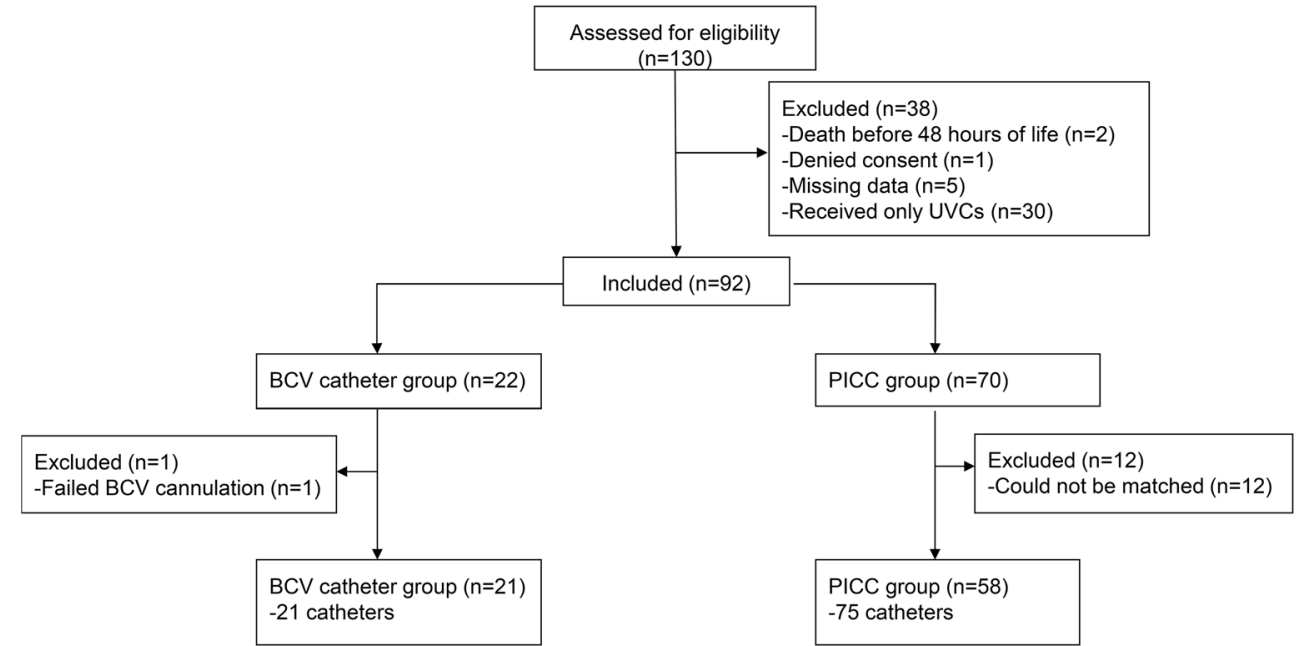


Table 2 Central venous catheter characteristics and outcomes

\begin{tabular}{llll}
\hline Characteristics & $\mathbf{B C V}(\boldsymbol{n}=\mathbf{2 1})$ & $\mathbf{E C C}(\boldsymbol{n}=\mathbf{7 5})$ & $\boldsymbol{p}$ \\
CVCs per infant & $1(1-1)$ & $2(1-4)$ & 0.044 \\
DOL at insertion & $12(9.5-15)$ & $8(4-10)^{\dagger}$ & 0.001 \\
Weight at insertion & $990(885-1058)$ & $980(760-1185)$ & 0.940 \\
Number of punctures & $1(1-3)$ & $2(1-5)$ & $<0.001$ \\
Number of punctures veins & $1(1-1)$ & $2(1-3)$ & 0.006 \\
CVC indication & & & \\
-Routine care & $1(4.7)$ & $66(88)$ & \\
-Surgery & $7(33.3)$ & $1(1.3)$ & $<0.001$ \\
-No other access & $6(38.6)$ & $1(1.3)$ & \\
-Shock/inotropics & $7(33.3)$ & $2(2.7)$ & \\
-Other reasons & $0(0)$ & $5(6.6)$ & \\
CVC withdrawal & & & \\
-End of treatment & $20(95.3)$ & $50(66.6)$ & \\
-Elective change & $0(0)$ & $4(5.3)$ & \\
-CLABSI & $1(4.7)$ & $11(14.7)$ & \\
-CVC dysfunction & $0(0)$ & $9(12)$ & \\
AB days per catheter & $14(12-16)$ & $5(2-8)$ & $<0.001$ \\
PN days per catheter & $13(8-18)$ & $6(3-9)$ & $<0.001$ \\
CVC days & $15(13-18)$ & $8(6-11)$ & $<0.001$ \\
Outcomes & $\mathbf{B C V ~ ( n = 2 1 )}$ & $\mathbf{P I C C ~ ( n = 7 5 )}$ & \\
CLABSI density rate & $3.05 / 1000$ & $21.1 / 1000$ & $<0.001$ \\
CLABSI per infant & $1(4.7)$ & $12(20.7)$ & 0.089 \\
CLABSI episodes & $1(4.7)$ & $14(18.6)$ & 0.121 \\
CLABSI/death & $3(14.2)$ & $17(29.3)$ & 0.202 \\
CLABSI pathogen & & & - \\
-CNS & 0 & 10 & \\
-Staphylococcus aureus & 0 & 1 & \\
-Enterococcus & 0 & 1 & \\
-Klebsiella & 1 & 1 & \\
-Serratia & 0 & & \\
\hline
\end{tabular}

Abbreviations: $A B$ parenteral antibiotics; $C V C$ central venous catheter; CLABSI catheter-related blood stream infection; $C N S$ coagulase-negative staphylococcus; $D O L$ day of life; $G A$ gestational age; $P N$ parenteral nutrition days. $p$ values for the $U$ Mann-Whitney test are shown. Data are summarized as median (IQR) except CVC per infant, number of veins punctured, and number of punctures that are summarized as median (range)

inotropic use, blood transfusion, hemodynamically significant PDA, NEC) than infants with ECCs.

The CVC-related outcomes are summarized in Table 2. Compared to ECC insertion, the first-attempt success rate of US-guided BCV cannulation was higher $(80.9 \%$ vs $42.6 \%$; $p<0.001$ ) (Fig. 2), and the number of punctured veins ((1 [1] vs $2[1-3] ; p=0.006)$ and puncture attempts ( $1[1]$ vs 2 $[1-3] ; p<0.001)$ were lower. Accidental arterial punctures and/or pneumothorax were not observed. BCV catheters were inserted later in postnatal life than were ECCs, but the infant's weight at the time of insertion was similar. Indications for

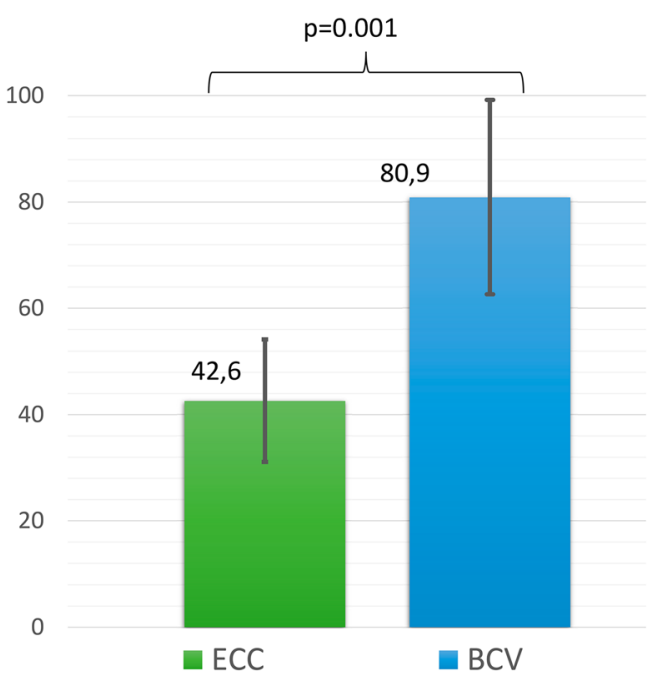

Fig. 2 Successful CVC placement at first puncture attempt. Abbreviations: $\mathrm{BCV}$, brachiocephalic vein; ECC, epicutaneous cava catheter. $Y$-axis indicates percentage with $95 \%$ confidence interval error bars

CVCs differed between the study groups: ECCs were used mostly for routine care (maintenance fluids, antibiotics, and parenteral nutrition), whereas BCVs were inserted in cases of high-risk surgery (laparotomy for NEC in 6 infants and gastric volvulus in one infant), hemodynamic instability requiring inotropics and invasive monitoring (PDA postligation syndrome in 4 infants and septic shock in 2 infants), and vein exhaustion (6 infants). Most BCVs were electively withdrawn after completion of treatment; CLABSI and catheter dysfunction were frequent reasons for nonelective removal of ECCs. The indwelling time of BCV catheters was significantly longer than that of PICCs [15 (13-18) days vs. 8 (6-11) days; $p<0.001$ ).

The CLABSI density rate was significantly lower for BCV catheters than ECCs (3.05/1000 CVC days vs. 21.1/1000 CVC days; $p<0.001)$. One of 21 infants $(4.7 \%)$ had one CLABSI

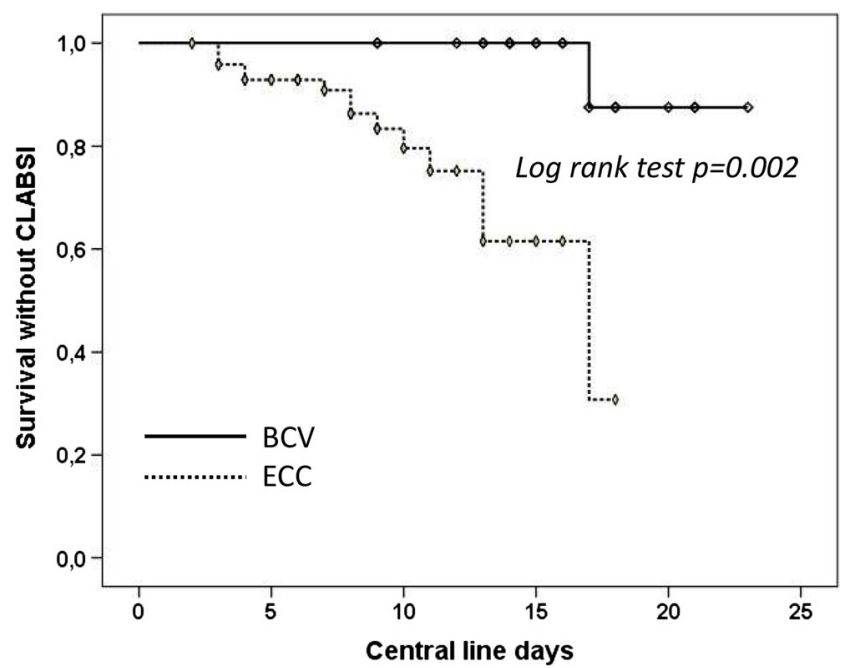

Fig. 3 Kaplan-Meier curve. Abbreviations: BCV, brachiocephalic vein; ECC, epicutaneous cava catheter 
Table 3 Cox regression analysis for the occurrence of CLABSI

Univariate analysis

\begin{tabular}{|c|c|c|c|c|c|}
\hline Dependent variable: CLABSI & Hazard ratio (CI 95\%) & $p$ & Dependent variable: CLABSI & Hazard ratio (CI 95\%) & $p$ \\
\hline $\mathrm{ECC}$ (vs BCV) & $14.2(1.8-117)$ & 0.013 & $\mathrm{ECC}$ (vs BCV) & $36(2.5-511)$ & 0.008 \\
\hline CVC placement attempts & $1.6(1.1-2.3)$ & 0.004 & CVC placement attempts & $1.8(1.2-2.7)$ & 0.004 \\
\hline RDS (surfactant) & $4.6(1.03-20.8)$ & 0.045 & RDS (surfactant) & $11.1(1.2-101)$ & 0.033 \\
\hline Antibiotic days during CVC & $0.87(0.776-0.97)$ & 0.017 & & & \\
\hline Weight at insertion (grams) & $0.998(0.995-1)$ & 0.099 & & & \\
\hline
\end{tabular}

Variables considered in the univariate analysis: type of catheter (ECC vs BCV) sex, gestational age at insertion, weight at insertion, cesarean delivery, CRIB score, 5 min Apgar score, respiratory distress syndrome (RDS), day of CVC insertion, parenteral nutrition days per CVC, antibiotics days per $\mathrm{CVC}$, transfusion of packed red blood cells during CVC permanence, and CVC placement attempts (number of punctures). Only variables with a $p$ value $<0.1$ in the univariate analysis are shown. Only variables that remained significant in the multivariate analysis are shown

episode with a BCV catheter, and 12 of 58 infants $(20.7 \%)$ had 14 episodes of CLABSI with ECCs $(p=0.089)$. CLABSI episodes per catheter $[1 / 21(4.7 \%)$ vs $14 / 75(18.6 \%), p=0.121]$ and CLABSI per infant or death $(3 / 21$ (14.25) vs. $17 / 58$ $(29.3 \%) ; p=0.202)$ were not different between the groups. Survival curves using the Kaplan-Meier method with the logrank test are shown in Fig. 3. CVC type (ECC vs BCV) was associated with increased CLABSI risk in Cox regression analysis after adjustment for weight at insertion time, respiratory distress syndrome, number of CVC placement attempts, and days of antibiotic therapy during CVC dwell time (hazard ratio 36; 95\% CI, 2.5-511; $p=0.008$ ) (Table 3).

\section{Discussion}

CLABSI is a major complication during the maintenance of CVCs and has an adverse impact on morbidity/mortality, health care costs, and neurological long-term outcomes of preterm infants $[15,16]$. The risk of CLABSI is greater in preterm infants than in older infants, children, and adults due to the unique vulnerability of immature infants, their prolonged CVC dwell times, and their high-intensity critical care needs [17]. Major efforts have been made to control risk factors and reduce the CLABSI-associated burden [18-20]. One aspect that might influence the CLABSI rate is the type of CVC and the insertion technique $[1,21]$. In this observational study, we found a significantly lower rate of CLABSI when US-guided supraclavicular BCV catheters were used compared to standard ECCs in preterm infants. Importantly, such a difference has been observed despite $\mathrm{BCV}$ catheters being used in higher-risk infants and staying in place for a longer period [22]. Seemingly, the observed reduction in CLABSI was driven by a reduced incidence of CLABSI episodes caused by CoNS. While mortality of SCN sepsis is low, the detrimental effects on neurologic development are substantial and similar to those of more virulent bacteria [23, 24]. Therefore, we consider our finding of clinical relevance.
Some previous research has suggested that percutaneous CVCs are associated with an increased risk of infection compared to ECCs in children, though the results among studies are contradictory $[25,26]$. In neonates, percutaneous CVCs are believed to carry a greater risk of CLABSI than ECCs, although supporting evidence is lacking [27]. Of note, no study has compared US-guided BCV cannulation with standard vascular access in preterm infants. One previous study by Habas et al. specifically assessed the risk of CLABSI with US-guided supraclavicular BCV cannulation against other CVC insertion sites (femoral, internal jugular vein, and infraclavicular subclavian vein) in older children in the pediatric intensive care unit (PICU), with the authors reporting a reduced CLABSI density rate $(\mathrm{BCV}, 2.8 / 1000$ days vs other-CVC, 8.96/1000 days; $p=$ 0.006) [28]. In a retrospective analysis, Biasucci D et al. observed a remarkable reduction in CLABSI from 15/1000 to 1.5/ 1000 days after the implementation of a CVC insertion bundle in the PICU [29]. The bundle included systematic assessment of central veins using the $\mathrm{RaCeVa}$ protocol and resulted in a radical shift in the choice of CVC insertion site in favor of the BCV (from 0 to $85 \%$ after bundle implementation) [30]. Other innovative elements in their bundle included routine tunneling of the catheter, sutureless fixation, glue sealing of the exit site, and specific simulation training; therefore, it is difficult to ascertain the exact contribution of BCV cannulation to the observed decrease in CLABSI.

The potential technical advantages of the US-guided supraclavicular approach for the BCV have been previously highlighted and include access to a large vein, fine control of needle advancement, a high first-pass cannulation success rate with few mechanical complications, and technical feasibility even in low-weight infants [31]. However, the reasons why this technique may reduce the risk of catheter colonization and infection are less obvious. Coagulation activation and the formation of a fibroblastic sleeve along the catheter interfere with biofilm formation, bacterial growth, and colonization of the extraluminal side of the catheter $[32,33]$. Additionally, the high first-attempt 
insertion success rate achieved with the US-guided supraclavicular approach might lead to less endothelial damage during insertion and reduced thrombogenesis. In fact, in our study, the number of puncture attempts was an independent risk factor for CLABSI. Moreover, blood flow stasis is a classic pathogenic determinant of thrombus formation; in this sense, among the veins amenable to percutaneous catheterization, the $\mathrm{BCV}$ is the largest and has the highest flow, which permits a high catheter-to-vein diameter ratio without flow disruption [34-36]. Exit sites in the supraclavicular area may also contribute to reducing contamination and avoiding site humidity, which facilitates easier adhesion of dressings [37]. In contrast to ECC lines, a large-bore CVC facilitates repeated blood sampling, avoiding peripheral venipunctures during catheter use, a factor that has been implicated in bacteremia and catheter colonization during the use of ECCs [38].

ECCs are often inserted after the removal of UVCs. These devices permit infusion rates up to $1-2 \mathrm{ml} / \mathrm{min}$, which are adequate for infusion of fluids, blood products, medications, or vasoactive drugs in most infants [39]. Nonetheless, ECCs do not permit the blood sampling, hemodynamic monitoring, multiple simultaneous infusions, or high-flow infusion rates that may be needed in critically ill preterm infants. Additionally, insertion failure rates are relatively high, and progression to a central position is hazardous even in experienced hands [40]. Catheter dysfunction and mechanical complications such as thrombosis and infiltration are relatively frequent, which often leads to nonelective removal [41].

If $\mathrm{BCV}$ cannulation is highly successful and catheter performance is superior to ECCs, why are percutaneous BCV catheters not inserted in most neonates who need central vein access when the umbilical vein is no longer available? We consider that there are some important limitations for the widespread use of BCV cannulation in preterm infants. The insertion of a percutaneous $\mathrm{CVC}$ through the $\mathrm{BCV}$ in a preterm is a challenging and delicate procedure. To be performed safely, it requires a deeply sedated and motionless infant, which is not always feasible. In addition, to be fully implemented, this approach would require around-the-clock availability of staff with sufficient expertise in US-guided cannulation. Finally, studies supporting $\mathrm{BCV}$ cannulation have been performed by highly experienced groups (pediatric anesthesiologists and vascular access teams) and have consisted of case series with near $100 \%$ insertion success rates but without any comparison to standard techniques; therefore, a publication bias may be a concern $[4,31]$. However, if our findings are confirmed in other studies, reduced CLABSI should be taken into account together with already known technical advantages in the decision to insert BCV catheters in preterm infants, and the indication of this new vascular access might be expanded.

Our study has some limitations. This is a single-center nonrandomized retrospective study that included a limited number of preterm infants; accordingly, our results should be considered a proof of concept and taken with caution. As all observational studies, there is an inherent risk of confounding bias. We tried to reduce this risk by selecting matched controls. However, a more refined approach would have been to include all patients and perform propensity score matching [42]; in our infant population, $\mathrm{BCV}$ cannulation was performed in selected infants by a unique experienced intensivist, whereas ECCs were inserted by a team of 10 nurses who might have heterogeneous levels of expertise [43]. Regardless, all nurses were certified neonatal practitioners and used the same protocol for ECC insertion. Infants with $\mathrm{BCV}$ catheters received more days of antibiotic therapy during CVC use, which might have had a protective effect on CLABSI and act as a confounder. However, the antibiotic protocol was common and the difference in antibiotic days coverage was driven by differences in indwelling time without an observable effect of antibiotics on CLABSI rate in the multivariate analysis. In addition, the CVC maintenance policy was the same for both types of catheters. Finally, as BCV catheters were inserted in patients in poorer condition and the indwelling duration was longer than that of ECCs, we think that there was no significant risk of bias favoring BCV catheters. Some aspects of our CVC insertion and maintenance policy are controversial. We used surgical suture for fixation of BCV catheters. This should be avoided whenever possible given that it may increase the risk of infection. Commercially available sutureless devices are a better option. In the same line, the use of low-dose heparin to maintain catheter patency is outdated and not supported by current scientific evidence [44]. Finally, our ECC CLABSI rate is relatively high compared to those reported by studies from other countries. For these reasons, our results may lack external validity [12, 22].

\section{Conclusions}

US-guided supraclavicular cannulation of the BCV in our very-low-birth-weight preterm infants was associated with a reduced incidence of CLABSI compared to standard ECCs. This fact should be taken into account in selected infants who need a large-bore CVC and should prompt additional multicenter prospective studies on the subject.

Authors' contributions IOE conceived and designed the study, analyzed the data, and wrote the first draft of the manuscript.

BAJ acquired the data and critically reviewed the manuscript.

$\mathrm{AFG}$ acquired the data and critically reviewed the manuscript.

SST acquired the data and critically reviewed the manuscript.

ARN conceived and designed the study and critically reviewed the manuscript.

All authors gave their approval to the final version of the manuscript and are responsible for the accuracy and integrity of the study.

\section{Compliance with ethical standards}

Conflict of interest The authors declare that they have no conflict of interest. 
Ethical approval The study protocol was reviewed and approved by the Institutional Review Board (IRB) and informed consent was obtained from all individual participants included in the study (parents or legal guardians provided informed consent for participation).

\section{References}

1. Shalabi M, Adel M, Yoon E, Aziz K, Lee S, Shah PS et al (2015) Risk of infection using peripherally inserted central and umbilical catheters in preterm neonates. Pediatrics 136:1073-1079

2. Pittiruti M (2013) Central venous catheters in neonates: old territory, new frontiers. Invited commentary to peripherally inserted central venous catheters in critically ill premature neonates, by Ozkiraz et al. J Vasc Access 14:320-324

3. Pittiruti M (2012) Ultrasound guided central vascular access in neonates, infants and children. Curr Drug Targets 13:961-969

4. Lausten-Thomsen U, Merchaoui Z, Dubois C, Eleni Dit Trolli S, Le Saché N, Mokhtari M et al (2017) Ultrasound-guided subclavian vein cannulation in low birth weight neonates. Pediatr Crit Care Med 18:172-175

5. Pirotte T, Veyckemans F (2007) Ultrasound-guided subclavian vein cannulation in infants and children: a novel approach. Br J Anaesth 98:509-514

6. Oulego-Erroz I, Alonso-Quintela P, Terroba-Seara S, JiménezGonzález A, Rodríguez-Blanco S, Vázquez-Martínez JL (2018) Ultrasound-guided cannulation of the brachiocephalic vein in neonates and preterm infants: a prospective observational study. Am J Perinatol 35:503-508

7. Menéndez JJ, Verdú C, Calderón B, Gómez-Zamora A, Schüffelmann C, de la Cruz JJ, de la Oliva P (2016) Incidence and risk factors of superficial and deep vein thrombosis associated with peripherally inserted central catheters in children. J Thromb Haemost 14:2158-2168

8. Sharp R, Cummings M, Fielder A, Mikocka-Walus A, Grech C, Esterman A (2015) The catheter to vein ratio and rates of symptomatic venous thromboembolism in patients with a peripherally inserted central catheter (PICC): a prospective cohort study. Int J Nurs Stud 52:677-685

9. Alonso-Quintela P, Oulego-Erroz I, Rodriguez-Blanco S, MuñizFontan M, Lapeña-López-de Armentia S, Rodriguez-Nuñez A (2015) Location of the central venous catheter tip with bedside ultrasound in young children: can we eliminate the need for chest radiography? Pediatr Crit Care Med 16:e340-e345

10. Monagle P, Chan AKC, Goldenberg NA, Ichord RN, Journeycake JM, Nowak-Göttl U, Vesely SK (2012) Antithrombotic therapy in neonates and children: antithrombotic therapy and prevention of thrombosis, 9th ed: American College of Chest Physicians Evidence-Based Clinical Practice Guidelines. Chest. 141(2 Suppl):e737S-e801S

11. García P, San Feliciano L, Benito F, García R, Guzmán J, Salas S et al (2013) Outcome at two years corrected age of a cohort of very low birth weight infants from hospitals within the neonatal SEN1500 network. An Pediatr 79:279-287

12. Madrid-Aguilar M, López-Herrera MC, Pérez-López J, EscuderoArgaluza J, Santesteban-Otazu E, Piening B et al (2019) Implementation of NeoKissEs in Spain: a validated surveillance system for nosocomial sepsis in very low birth weight infants. An Pediatr 91:3-12

13. Turner D, Hammerman C, Rudensky B, Schlesinger Y, Goia C, Schimmel MS (2008) Procalcitonin in preterm infants during the first few days of life: introducing an age related nomogram. Arch Dis Child Fetal Neonatal Ed 91:F283-F286

14. Horan TC, Andrus M, Dudeck MA (2008) CDC/NHSN surveillance definition of health care-associated infection and criteria for specific types of infections in the acute care setting. Am J Infect Control 36:309-332

15. Zonnenberg IA, van Dijk-Lokkart EM, van den Dungen FAM, Vermeulen RJ, van Weissenbruch M (2019) Neurodevelopmental outcome at 2 years of age in preterm infants with late-onset sepsis. Eur J Pediatr 178:673-680

16. Shah DK, Doyle LW, Anderson PJ, Bear M, Daley AJ, Hunt RW, Inder TE (2008) Adverse neurodevelopment in preterm infants with postnatal sepsis or necrotizing enterocolitis is mediated by white matter abnormalities on magnetic resonance imaging at term. $\mathrm{J}$ Pediatr 153:170-175

17. Balkhy HH, Zingg W (2014) Update on infection control challenges in special pediatric populations. Curr Opin Infect Dis 27: 370-378

18. Schulman J, Stricof R, Stevens TP, Horgan M, Gase K, Holzman IR, Koppel RI, Nafday S, Gibbs K, Angert R, Simmonds A, Furdon SA, Saiman L, New York State Regional Perinatal Care Centers (2011) Statewide NICU central-line-associated bloodstream infection rates decline after bundles and checklists. Pediatrics 127:436-444

19. Advani SD, Murray TS, Murdzek CM, Aniskiewicz MJ, Bizzarro MJ (2020) Shifting focus toward healthcare-associated bloodstream infections: the need for neonatal intensive care unit-specific NHSN definitions. Infect Control Hosp Epidemiol 41:181-186

20. Wilder KA, Wall B, Haggard D, Epperson T (2016) CLABSI reduction strategy: a systematic central line quality improvement initiative integrating line-rounding principles and a team approach. Adv Neonatal Care 16:170-177

21. Arvaniti K, Lathyris D, Blot S, Apostolidou-Kiouti F, Koulenti D, Haidich A-B (2017) Cumulative evidence of randomized controlled and observational studies on catheter-related infection risk of central venous catheter insertion site in ICU patients: a pairwise and network meta-analysis. Crit Care Med 45:e437-e448

22. Greenberg RG, Cochran KM, Smith PB, Edson BS, Schulman J, Lee $\mathrm{HC}$ et al (2015) Effect of catheter dwell time on risk of central line-associated bloodstream infection in infants. Pediatrics 136: 1080-1086

23. Dong Y, Speer CP (2015) Late-onset neonatal sepsis: recent developments. Arch Dis Child Fetal Neonatal Ed 100:F257-F263

24. Stoll BJ, Hansen NI, Adams-Chapman I, Fanaroff AA, Hintz SR, Vohr B et al (2004) Neurodevelopmental and growth impairment among extremely low-birth-weight infants with neonatal infection. JAMA 292:2357-2365

25. Yamaguchi RS, Noritomi DT, Degaspare NV, Muñoz GOC, Porto APM, Costa SF, Ranzani OT (2017) Peripherally inserted central catheters are associated with lower risk of bloodstream infection compared with central venous catheters in paediatric intensive care patients: a propensity-adjusted analysis. Intensive Care Med 43: 1097-1104

26. Noonan PJ, Hanson SJ, Simpson PM, Dasgupta M, Petersen TL (2018) Comparison of complication rates of central venous catheters versus peripherally inserted central venous catheters in pediatric patients. Pediatr Crit Care Med 19:1097-1105

27. Soares BN, Pissarra S, Rouxinol-Dias AL, Costa S, Guimarães H (2018) Complications of central lines in neonates admitted to a level III neonatal intensive care unit. J Matern Fetal Neonatal Med 31: 2770-2776

28. Habas F, Baleine J, Milési C, Combes C, Didelot M-N, RomanoBertrand S, Grau D, Parer S, Baud C, Cambonie G (2018) Supraclavicular catheterization of the brachiocephalic vein: a way to prevent or reduce catheter maintenance-related complications in children. Eur J Pediatr 177:451-459

29. Biasucci DG, Pittiruti M, Taddei A, Picconi E, Pizza A, Celentano D, Piastra M, Scoppettuolo G, Conti G (2018) Targeting zero catheter-related bloodstream infections in pediatric intensive care unit: a retrospective matched case-control study. J Vasc Access 19:119-124 
30. Spencer TR, Pittiruti M (2019) Rapid central vein assessment (RaCeVA): a systematic, standardized approach for ultrasound assessment before central venous catheterization. J Vasc Access 20: 239-249

31. Merchaoui Z, Lausten-Thomsen U, Pierre F, Ben Laiba M, Le Saché N, Tissieres P (2017) Supraclavicular approach to ultrasound-guided brachiocephalic vein cannulation in children and neonates. Front Pediatr 5:211

32. Hill S, Hamblett I, Brady S, Vasileukaya S, Zuzuarregui I, Martin F (2019) Central venous access device-related sheaths: a predictor of infective and thrombotic incidence? Br J Nurs 28:S10-S18

33. Gominet M, Compain F, Beloin C, Lebeaux D (2017) Central venous catheters and biofilms: where do we stand in 2017? Acta Pathol Microbiol Immunol Scand 125:365-375

34. Barone G, D'Andrea V, Vento G, Pittiruti M (2019) A systematic ultrasound evaluation of the diameter of deep veins in the newborn: results and implications for clinical practice. Neonatology 115:335-340

35. Male C, Chait P, Andrew M, Hanna K, Julian J, Mitchell L et al (2003) Central venous line-related thrombosis in children: association with central venous line location and insertion technique. Blood 101:4273-4278

36. Salim MA, DiSessa TG, Arheart KL, Alpert BS (1995) Contribution of superior vena caval flow to total cardiac output in children. A Doppler echocardiographic study. Circulation 92: 1860-1865

37. Timsit J-F, Bouadma L, Ruckly S, Schwebel C, Garrouste-Orgeas M, Bronchard R, Calvino-Gunther S, Laupland K, Adrie C, Thuong M, Herault MC, Pease S, Arrault X, Lucet JC (2012) Dressing disruption is a major risk factor for catheter-related infections. Crit Care Med 40:1707-1714

38. Cheng H-Y, Lu C-Y, Huang L-M, Lee P-I, Chen J-M, Chang L-Y (2016) Increased frequency of peripheral venipunctures raises the risk of central-line associated bloodstream infection in neonates with peripherally inserted central venous catheters. J Microbiol Immunol Infect 49:230-236

39. Westergaard B, Classen V, Walther-Larsen S (2013) Peripherally inserted central catheters in infants and children - indications, techniques, complications and clinical recommendations. Acta Anaesthesiol Scand 57:278-287

40. Al Hamod DA, Zeidan S, Al Bizri A, Baaklini G, Nassif Y (2016) Ultrasound-guided central line insertion and standard peripherally inserted catheter placement in preterm infants: comparing results from prospective study in a single-center. North Am J Med Sci 8: 205-209

41. Erhard DM, Nguyen S, Guy KJ, Casalaz DM, König K (2017) Dwell times and risk of non-elective removal of 1-French peripherally inserted central catheters according to catheter tip position in very preterm infants. Eur J Pediatr 176:407-411

42. Zhang Z (2017) Propensity score method: a non-parametric technique to reduce model dependence. Ann Transl Med 5:7

43. Legemaat MM, Jongerden IP, van Rens RMFPT, Zielman M, van den Hoogen A (2015) Effect of a vascular access team on central line-associated bloodstream infections in infants admitted to a neonatal intensive care unit: a systematic review. Int J Nurs Stud 52: $1003-1010$

44. López-Briz E, Ruiz Garcia V, Cabello JB, Bort-Martí S, Carbonell Sanchis R, Burls A (2018) Heparin versus $0.9 \%$ sodium chloride locking for prevention of occlusion in central venous catheters in adults. Cochrane Database Syst Rev 7:CD008462

Publisher's note Springer Nature remains neutral with regard to jurisdictional claims in published maps and institutional affiliations. 